\title{
Teamworking in endoscopy: a human factors toolkit for the COVID-19 era
}

\author{
Authors \\ Srivathsan Ravindran ${ }^{1,2}$, Manmeet Matharoo ${ }^{3}$, Mark Coleman ${ }^{1,4}$, Sarah Marshall ${ }^{3,5}$, Chris Healey ${ }^{1,6}$, Ian Penman ${ }^{7,8}$, \\ Siwan Thomas-Gibson ${ }^{2,3}$
}

Institutions

1 Joint Advisory Group on Gastrointestinal Endoscopy, Royal College of Physicians, London, United Kingdom

2 Department of Surgery and Cancer, Imperial College London, United Kingdom

3 Wolfson Unit for Endoscopy, St Mark's Hospital and Academic Institute, London, United Kingdom

4 Department of Colorectal Surgery, University Hospitals Plymouth NHS Trust, Plymouth, United Kingdom

5 Nurses Association Committee, British Society of Gastroenterology, London, United Kingdom

6 Department of Gastroenterology, Airedale NHS Foundation Trust, Keighley, United Kingdom

7 Centre for Liver and Digestive Disorders, Royal Infirmary of Edinburgh, Edinburgh, United Kingdom

8 Endoscopy Section Committee, British Society of Gastroenterology, London, United Kingdom

submitted 7.6.2020

accepted after revision 22.6.2020

published online 22.6.2020

\section{Bibliography}

Endoscopy 2020; 52: 879-883

DOI 10.1055/a-1204-5212

ISSN 0013-726X

(c) 2020. Thieme. All rights reserved. Georg Thieme Verlag KG, Rüdigerstraße 14, 70469 Stuttgart, Germany

Supplementary material

Online content viewable at:

https://doi.org/10.1055/a-1204-5212
丹 Scan this QR-Code for the author commentary.

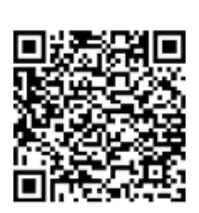

Corresponding author

Srivathsan Ravindran, MBBS BSc MSc, Joint Advisory Group on Gastrointestinal Endoscopy, Royal College of Physicians,

11 St Andrews Place, London NW1 4LE, United Kingdom Fax: +44-20-30751649

sravindran1@nhs.net

\section{ABSTRACT}

Background Endoscopy services have had to rapidly adapt their working practices in response to COVID-19. As recovery of endoscopy services proceeds, our workforce faces numerous challenges that can impair effective teamworking. We designed and developed a novel toolkit to support teamworking in endoscopy during the pandemic.

Methods A human factors model was developed to understand the impact of COVID-19 on endoscopy teams. From this, we identified a set of key teamworking goals, which informed the development of a toolkit to support several team processes. The toolkit was refined following expert input and refinement over a 6-week period.

Results The toolkit consists of four cognitive aids that can be used to support team huddles, briefings, and debriefs, alongside techniques to optimize endoscopic nontechnical skills across the patient-procedure pathway. We describe the processes that local endoscopy units can employ to implement this toolkit.

Conclusion A toolkit of cognitive aids, based on human factors principles, may be useful in supporting teams, helping them adapt to working safely in the era of COVID-19.

\section{Introduction}

Within the space of a few months, COVID-19 has had an unprecedented effect on our healthcare systems internationally. Elective endoscopy within the UK had all but stopped as a result of challenges faced early on in the pandemic [1]. We now face the challenge of recovering endoscopy services, and it is almost certain that working practices will change for the foreseeable future.

Guidance from the British Society of Gastroenterology (BSG) describes strategies for recovery, including the development of "COVID-minimized" endoscopy units [2,3]. This is further supported by guidance from the Joint Advisory Group on Gastrointestinal Endoscopy (JAG) and the European Society of Gastroin- 


\begin{tabular}{l} 
Input \\
Patient \& Healthcare Professional \\
factors \\
- Anxiety/stress/fatigue/illness \\
- Inexperience/lack of knowledge \\
- Fear of infection \\
Task factors \\
- Changes to workflow \\
- Changes to patient-healthcare \\
professional interaction \\
- Greater task complexity \\
Tool factors \\
- Personal protective equipment \\
(PPE) \\
Environmental factors \\
- Change to working environ- \\
ment (physical layout/space) \\
\hline
\end{tabular}

Process
Physical
- Heat
- Dehydration
- Exhaustion
Cognitive
- Reduced perception of situation
or environment
- Reduced hearing
- Reduced attention
- Impaired reasoning
Social
- Impaired verbal and non-verbal
communication
- Low motivation

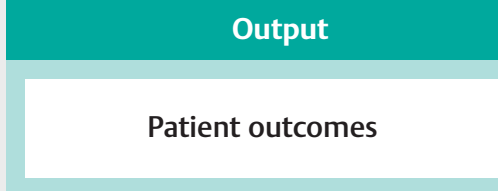

Organisational outcomes

Cognitive

Reduced perception of situation

ced attention

Social

Impaired verbal and non-verba communication

- Low motivation

- Fig. 1 Input, process, and output model of factors influencing endoscopy team performance following COVID-19.

testinal Endoscopy (ESGE) $[4,5]$. As a result, key changes to practice are being undertaken, including patient screening, room management, and use of personal protective equipment (PPE). However, COVID-19 also presents challenges to our workforce, who will have to adapt to novel environments and practices. Endoscopy teams will also be re-forming after periods of redeployment, potentially anxious about the conceivable ongoing personal risk to themselves and their families.

Effective teamworking is crucial to team performance and, ultimately, to patient outcomes [6]. Adaptations to practice are evolving rapidly as new guidance is released or updated. Teams need to be supported in developing flexibility and the additional situational demand that results. This article outlines practical steps to enhance teamworking in endoscopy during COVID-19 through the use of a novel toolkit.

\section{Methods}

\section{Developing the Endoscopy Team Toolkit}

Healthcare systems can be defined by a human factors model of inputs, processes, and outcomes [7]. Understanding how this model is influenced by the pandemic allows us to design and implement appropriate interventions to improve patient and organizational outcomes. Endoscopic nontechnical skills (ENTS) include the communication, teamwork, leadership, situational awareness, and judgment fundamental to performing endo- scopic procedures [8] and should be considered during the design process. A human factors model for endoscopy during COVID-19 was therefore developed ( $\mathbf{F i g} \mathbf{1}$ ), which informed a set of key goals for endoscopy teams, as presented below.

1. Planning and anticipating problems

2. Optimizing communication

3. Fostering a sense of team cohesion

4. Flattening hierarchy

5. Sharing task burden

6. Providing support and wellbeing where needed.

The goals listed informed the design and development of a toolkit to support endoscopy teams, complementing the current BSG, JAG, and ESGE guidance for endoscopy services [25]. The toolkit consists of four user-friendly cognitive aids that operationalize teamworking processes, supporting a system of team briefing and debriefing. Input from experts in human factors (including the Clinical Human Factors Group [9]), nontechnical skills, and patient safety was incorporated, supported by nursing and clinical oversight. This intervention was designed and implemented promptly in response to clinical need, and refinements were made following a 6-week period of testing and user feedback at a tertiary endoscopy unit. A handbook of the toolkit aids, including descriptors, can be found in the online supplementary material. We describe the toolkit in more detail in the sections that follow. 


\section{A Whole team huddle}

Planning for case management for the day ahead

Location \& time: e.g. Endoscopy recovery 8:00 am

Team present: e.g. Endoscopy consultant, endoscopy registrar, lead nurse, recovery nurses, decon staff

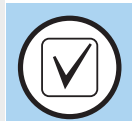

Team check-in

- Introductions \& greeting

- Health-check
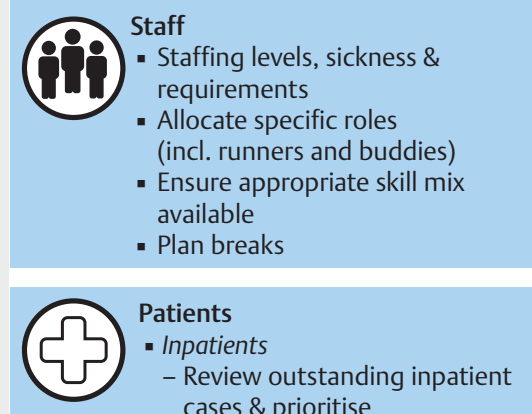

Patients

- Inpatients

- Review outstanding inpatient cases \& prioritise

- Arrange cases by COVID status (or other infectious disease)

- Alerting specialties if backup required

- Outpatients

- Identify lists running and timings

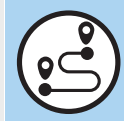

Flow

- Outline clinical setting for cases

- Contact parent team

- Convey anticipated running order to relevant teams

Equipment

- Specific alerts for endoscopes/ other equipment

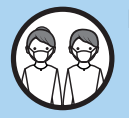

- Ensure appropriate stocks of PPE - Ensure stocks of cleaning/ disinfectant materials
B List team briefing

Preparing for the list and cases ahead Endoscopist, assistants, room runner

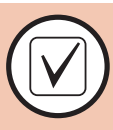

Team check-in

- Introductions \& greetings

- Health-check

- Encourage open discussion

Team members
- Names
- Allocate specific roles
- Review ENTS plan
(see ENTS in PPE aid)

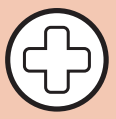

Case planning

- Review the case(s)

- Discuss the plan

- Identify \& anticipate problems

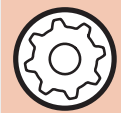

Equipment

- Identify \& source equipment

- Clarify essential and standby equipment

- Equipment check

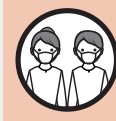

PPE

- Review donning and doffing

procedures

- Clarify and specific PPE requirements

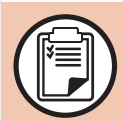

Checklist

- Complete the relevant checklist

elements outside of room

(if needed)

- Ensure adequate patient communication and consent confirmed

\section{List team debrief}

Learn from experiences and support team members

After each case and/or list

Describe
What
happened? $\rightarrow \begin{gathered}\text { Analyse } \\ \text { Why did it } \\ \text { happen? }\end{gathered} \rightarrow \begin{gathered}\text { Apply } \\ \text { What can we } \\ \text { do next time? }\end{gathered}$

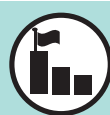

Outcomes

What did we achieve today?

- What worked well?

-What can we improve upon?

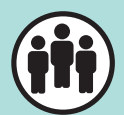

Teamworking

- Team dynamics

- Communication

- Roles and responsibilities

- Leadership

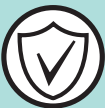

Safety

- Review and safety issues

- Incident reporting

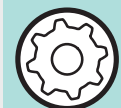

Equipment

- Equipment issues or faults

- Stock replenishment

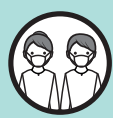

PPE

- Donning/doffing experiences

- PPE issues

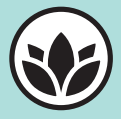

Wellbeing

- Check-in with all team members

- Review performance-limiting factors

- Signpost to relevant resources

- Fig.2 Endoscopy team toolkit: whole team huddle aid [A]; list team briefing [B]; list team debrief aid [C].

\section{Results}

\section{Pre-endoscopy huddle and briefing}

The terms "huddle" and "briefing" are often used synonymously to describe a meeting of team members before a task is performed; such meetings have been demonstrated to improve safety-related outcomes and team performance measures [10, 11]. Although these processes may already be embedded within endoscopy units, they should be adapted to the current situation in order to optimize teamworking and preserve patient safety.

Briefings familiarize team members with each other, allow task planning, and enhance communication. They can improve the quality of information sharing, promote accountability, empower team members, and provide a sense of team cohesion [12]. From a human factors perspective, briefings can be invaluable in flattening hierarchy - reducing the authority gradient between "senior" and "junior" staff by encouraging contributions from all multidisciplinary team members, thus improving safety. Aligning team goals, checking shared understanding, and providing the opportunity to share concerns are important in optimizing teamworking. For the purpose of the toolkit, we use the term "huddle" to describe a whole team huddle at the start of the day and "briefing" for the smaller team briefing prior to case and/or list. 


\section{Endoscopic Non-Technical Skills (ENTS) in PPE}

Tips to optimise ENTS in PPE (for in-room team and dedicated runner)

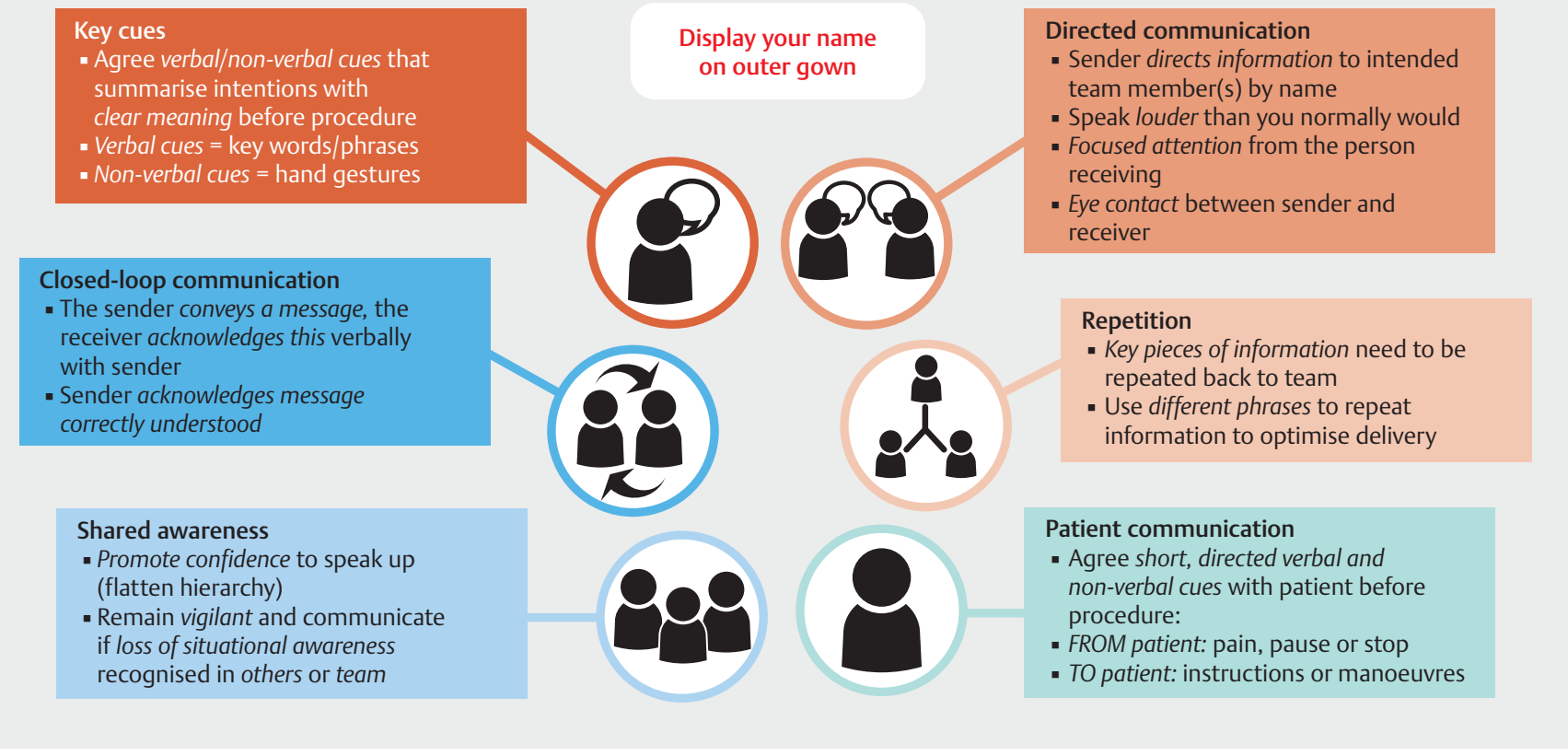

Fig.3 Endoscopy team toolkit aid for optimizing endoscopic nontechnical skills (ENTS) in personal protective equipment (PPE).

\section{Whole team huddle}

The whole team huddle describes a daily briefing with all endoscopy staff, including endoscopists, nurses, healthcare assistants, decontamination staff, and porters ( $\triangleright$ Fig. 2 a; further detail in the supplementary material). This is an opportunity to touch base with all staff members and to foster a positive teamworking culture.

\section{List team briefing}

For the core team running an endoscopy list (endoscopist, assistants, room runner), a more dedicated briefing is required, known as the "list team briefing" ( $>$ Fig. 2 b; further detail in the supplementary material). This builds on the principles of the whole team huddle with more specific, case-centered communication. The briefing should precede and complement the endoscopy safety checklist and is not a replacement for it. This is an opportunity to enhance the team-building process, encourage open contribution, flatten hierarchy, and develop a shared understanding [12].

\section{Team debrief}

Debriefing allows the team to collate, process, and act on information derived from a patient encounter to influence future behaviors and team performance. This process also strengthens team bonds by promoting interaction between members [13]. Ideally, all team members should be encouraged to contribute to the debrief in open discussion. The debrief aid provides a framework for these discussions (\$Fig. $2 c$ ). Debriefs should be led by a team member following a basic model of "description, analysis, and application" [14]. Team members describe what happened, consider the reasoning behind this, and offer solutions if needed. "Active" listening should be encouraged, enabling all team members to contribute and improving the effectiveness of idea generation. The COVID-19 pandemic has almost certainly amplified previous stressors and created new concerns in our workforce, and the debrief is a good opportunity to signpost wellbeing resources. Elements of the list team debrief are described in detail in the supplementary material.

\section{Optimizing ENTS in PPE}

A significant change to practice is the use of PPE during endoscopic procedures. At the time of writing, the choice of PPE (standard or enhanced) is governed by procedural type and patient risk stratification, including COVID-19 screening [2]. PPE may affect elements of procedural delivery, most notably ENTS. Both verbal and nonverbal communication may be impaired through use of respirator masks and head coverings [15]. These challenges should be anticipated prior to each case and highlighted in the team briefing as described previously. Team members should consider the following points to optimize communication ( $\mathbf{F i g . 3}$; further detail in the supplementary material):

- key cues

- directed communication

- closed-loop communication

- repetition. 
PPE may also impair effective patient communication, which may be exacerbated by language difficulties, auditory or visually impairment. Short, directed cues should be agreed with the patient in advance to convey issues such as pain or to pause or stop a procedure. Equally, instructions and procedural progress should be succinctly verbalized or gestured by team members to the patient. Situational awareness, and the ability to perceive, comprehend, and anticipate events, may also be compromised when PPE is in use. Team members should remember to remain vigilant at all times and be encouraged to speak up if they recognize a loss of situational awareness in other team members.

\section{Discussion}

The initial implementation of briefings and debriefings may be perceived as "added work" owing to the change to workflow needed to accommodate them [11]. Leadership from medical, nursing, and management teams is required to embed these changes from the outset. Benefits will be seen if staff are engaged in the process early on, recognizing that their involvement is valued with ongoing modifications tailored to their working needs. The toolkit may be used initially in a "checkliststyle" fashion, but as endoscopy teams become more familiar with the process, it can become more of an aide memoire. Some elements may appear repetitive; however, these processes can be adapted to suit local needs, and need not be exhaustively adhered to. Cognitive aids can be tailored further, followed by periods of testing and implementation.

To maintain consistency, a standardized time and location for the whole team huddle should be defined. It is important to set a time limit in order to focus discussion and minimize perceived disruptions to workflow. To promote engagement, the huddle lead can be rotated among medical and nursing staff. Huddles should adhere to social distancing principles and be supported by video conferencing if it is difficult to accommodate all staff members in a single space. Huddle discussions should be visually represented within a confidential area in the department, for example a whiteboard in staff areas, to provide a brief description of outcomes and engage staff members who cannot attend. Case briefs and debriefs should be short and succinct to facilitate workflow, whereas more time should be allocated for list debriefs to focus on team and procedural outcomes.

\section{Conclusions}

Endoscopy teams will continue to adapt to changes in practice precipitated by the COVID-19 pandemic. A toolkit of cognitive aids, based on human factors principles, may be useful in supporting this adjustment and will be helpful in the long term.

\section{Acknowledgments}

We would like to acknowledge the support of the Clinical Human Factors Group, particularly Jo Simmons and John Pickles, who helped to refine the toolkit aids.

\section{Competing interests}

The authors declare that they have no conflicts of interest.

\section{References}

[1] Edwards C, Penman ID, Coleman M. Gastrointestinal endoscopy during COVID-19: when less is more. Frontline Gastroenterol 2020; 11: 256-257

[2] Penman I, Edwards C, McKinlay A. British Society of Gastroenterology guidance on recommencing gastrointestinal endoscopy in the deceleration and early recovery phases of COVID-19 pandemic. British Society of Gastroenterology 2020: https://www.bsg.org.uk/covid-19advice/bsg-guidance-on-recommencing-gi-endoscopy-in-the-deceleration-early-recovery-phases-of-the-covid-19-pandemic/ (Accessed: 7 June 2020)

[3] Hayee B, Thoufeeq M, Rees C] et al. Safely restarting Gl endoscopy in the era of COVID-19. Gut 2020: doi:10.1136/gutjnl-2020-321688

[4] Joint Advisory Group on Gastrointestinal Endoscopy. Supplementary environment guidance following the COVID-19 pandemic. Joint Advisory Group on Gastrointestinal Endoscopy 2020: https://www.thejag. org.uk/covid-environment-guidance (Accessed: 7 June 2020)

[5] Gralnek IM, Hassan C, Beilenhoff U et al. ESGE and ESGENA Position Statement on gastrointestinal endoscopy and the COVID-19 pandemic. Endoscopy 2020; 52: 483-490

[6] Schmutz J, Manser T. Do team processes really have an effect on clinical performance? A systematic literature review $\mathrm{Br}$ J Anaesth 2013; 110: 529-544

[7] Karsh BT, Holden RJ, Alper S] et al. A human factors engineering paradigm for patient safety: designing to support the performance of the healthcare professional. Qual Saf Health Care 2006; 15: (Suppl. 01): i59-i65

[8] Ravindran S, Haycock A, Woolf K et al. Development and impact of an endoscopic non-technical skills (ENTS) behavioural marker system. BMJ Simul Technol Enhanc Learn 2020: doi:10.1136/bmjstel-2019000526

[9] The Clinical Human Factors Group. Key Human Factors messages when working under pressure. Clinical Human Factors Group 2020: https://chfg.org/what-are-clinical-human-factors/ (Accessed 7 June 2020)

[10] Franklin BJ, Gandhi TK, Bates DW et al. Impact of multidisciplinary team huddles on patient safety: a systematic review and proposed taxonomy. BMJ Qual Saf 2020: doi:10.1136/bmjqs-2019-009911

[11] Hicks CW, Rosen M, Hobson DB et al. Improving safety and quality of care with enhanced teamwork through operating room briefings. JAMA Surg 2014; 149: 863-868

[12] Goldenhar LM, Brady PW, Sutcliffe KM et al. Huddling for high reliability and situation awareness. BMJ Qual Saf 2013; 22: 899

[13] Salas E, Klein C, King $\mathrm{H}$ et al. Debriefing medical teams: 12 evidencebased best practices and tips. Jt Comm J Qual Patient Saf 2008; 34 : 518-527

[14] Jaye P, Thomas L, Reedy G. 'The Diamond': a structure for simulation debrief. Clin Teach 2015; 12: 171-175

[15] Ellis R, Hay-David AGC, Brennan PA. Operating during the COVID-19 pandemic: how to reduce medical error. Br J Oral Maxillofac Surg 2020; 58: 577-580 